\title{
Exact Numerical Solution of the BCS Pairing Problem
}

\author{
Feng Xu, An Min Wang, and Xiao-Dong Yang, and Hao You \\ Department of Modern Physics, University of Science and Technology of China, Hefei, 230026, P.R.China
}

\begin{abstract}
We propose a new simulation computational method to solve the reduced BCS Hamiltonian based on spin analogy and submatrix diagonalization. Then we further apply this method to solve superconducting energy gap and the results are well consistent with those obtained by Bogoliubov transformation method. The exponential problem of $2^{N}$-dimension matrix is reduced to the polynomial problem of $N$-dimension matrix. It is essential to validate this method on a real quantum computer and is helpful to understand the many-body quantum theory.
\end{abstract}

PACS numbers: 74.20.Fg, 03.67.Lx

BCS theory $\underline{\underline{1}}$ and its subsequent extension is a well established theory to explain the mechanism of superconducting property. With two gross simplifications: the free electron approximation and the effective interaction approximation 2,3 , a simplified BCS model is obtained and described by the reduced BCS Hamiltonian. There has been much work on solving this Hamiltonian. The mean field method is exact in the limit of large number of electrons where fluctuation can be neglected but disabled in the case of small number of electrons. Since Richardson's work ${ }^{4}$ in the 60's to now, the exactly solvable BCS Hamiltonian attracts much attention in connection with the problems in different areas of physics such as superconductivity, nuclear physics, physics of ultrasmall metallic grains.

Recently in $L .-A$. $W u$ et al.'s paper ${ }^{\underline{5}}$ an NMR experiment scheme performing a polynomial-time simulation of pairing model was reported. Based on this work we propose an explicit theory method to diagonalize the reduced BCS Hamiltonian through the spin analogy and submatrix diagonalization. Compared with the conventional method it is more useful in solving practical problem. The problem is solved in the spin space, which is convenient related to the qubit system. It gives a senseful alive method, quantum simulation, instead of the numerical diagonalization calculation. And it shows the potential to solve many-body problem by quantum computer. In fact more and more people concentrate on the research of simulating other physics systems by quantum computer ${ }^{6}$. The experimental quantum simulations about quantum harmonic oscillator ${ }^{7}$, three-spin artifical Hamiltonian ${ }^{8}$ and migration of excitation in a one-dimensional chain ${ }^{9}$ et al. have been realized. Recently a relative experiment is performed to get the eigenvalues of the BCS Hamiltonian through selecting a proper initial state and realizing Hamiltonian evolution. 10? $^{2}$

The exact solvable model, i.e. the reduced BCS Hamiltonian considered in this paper is $\frac{3}{2}, \underline{5}, 12$ :

$$
H_{\mathrm{BCS}}=\sum_{m=1}^{N} \frac{\left(\varepsilon_{m}-\varepsilon_{F}\right)}{2}\left(n_{m}+n_{-m}\right)-V \sum_{m, l=1}^{N} c_{m}^{\dagger} c_{-m}^{\dagger} c_{-l} c_{l}
$$

where $n_{ \pm m} \equiv c_{ \pm m}^{\dagger} c_{ \pm m}$ are the electron number operators, $c_{m}^{\dagger}\left(c_{m}\right)$ is the fermionic creation (annihilation) operator. The coupling coefficient is simplified as a constant $V^{2}, \underline{13}$. Note that the summation indexes $m=1,2, \cdots, N$ represent all of relevant quantum numbers, and the electron pairs are labelled by the the quantum number $m$ and $-m$, according to the Cooper pair situation where the paired electrons have equal energies but opposite momenta and spins: $m=$ $(\vec{k}, \uparrow)$ and $-m=(-\vec{k}, \downarrow)$. Introduce the pair creation operator $b_{m}^{\dagger}=c_{m}^{\dagger} c_{-m}^{\dagger}$ and the pair annihilation operator $b_{m}=c_{-m} c_{m}$. So one can write the Hamiltonian (1) as ${ }^{14}$ :

$$
H_{\mathrm{BCS}}=\sum_{m=1}^{N} \frac{\xi_{m}}{2}\left(n_{m}+n_{-m}\right)-V \sum_{m, l=1}^{N} b_{m}^{\dagger} b_{l}
$$

where $\xi_{m}=\varepsilon_{m}-\varepsilon_{F}$ is the free electron kinetic energy from Fermi surface ( $\varepsilon_{F}$ is the Fermi energy). There are two possible cases for every pair state $m$ : "occupation" and "empty", which are denoted respectively by:

$$
\chi_{1}=\left(\begin{array}{l}
1 \\
0
\end{array}\right), \quad \chi_{0}=\left(\begin{array}{l}
0 \\
1
\end{array}\right)
$$

where the spin up state $\chi_{1}$ indicates "occupation" and the spin down state $\chi_{0}$ indicates "empty". Obviously, $\frac{1}{2}\left(\sigma_{x}-\right.$ $\left.i \sigma_{y}\right) \chi_{1}=\chi_{0}$ and $\frac{1}{2}\left(\sigma_{x}-i \sigma_{y}\right) \chi_{0}=0$, then we can get the so-called spin-analogy corresponding of the pair annihilation 
operator $b_{m}$ as

$$
b_{m} \Rightarrow\left(\begin{array}{ll}
0 & 0 \\
1 & 0
\end{array}\right)=\frac{1}{2}\left(\sigma_{x}^{(m)}-i \sigma_{y}^{(m)}\right)=\sigma_{m}^{-}
$$

In the same way, the spin-analogy corresponding of the pair creation operator $b_{m}^{\dagger}$ becomes

$$
b_{m}^{\dagger} \Rightarrow\left(\begin{array}{ll}
0 & 1 \\
0 & 0
\end{array}\right)=\frac{1}{2}\left(\sigma_{x}^{(m)}+i \sigma_{y}^{(m)}\right)=\sigma_{m}^{+}
$$

From the pair number operator $n_{m}+n_{-m}$ has the eigenvalue 2 (which represents the electron number in every Cooper pair) when operating on $\chi_{1}$, and 0 when operating on $\chi_{0}$, it follows that

$$
n_{m}+n_{-m} \Rightarrow\left(\begin{array}{ll}
2 & 0 \\
0 & 0
\end{array}\right)=1+\sigma_{z}^{(m)}
$$

In fact, the fermionic pair operators satisfy the commutation algebra: $\operatorname{sl}(2)=\left\{b_{m}, b_{m}^{\dagger}, n_{m}+n_{-m}-1\right\}$, i.e. $s l(2)=$ $\left\{\sigma_{m}^{-}, \sigma_{m}^{+}, \sigma_{m}^{z}\right\}$. From formulas (4)-(6) one can express $H_{\mathrm{BCS}}$ in terms of the spin operators:

$$
\begin{aligned}
H_{\text {spin }} & \Rightarrow \sum_{m=1}^{N} \frac{\xi_{m}}{2}\left(1+\sigma_{z}^{(m)}\right)-V \sum_{m, l=1}^{N} \frac{1}{2}\left(\sigma_{x}^{(m)}+i \sigma_{y}^{(m)}\right) \frac{1}{2}\left(\sigma_{x}^{(l)}-i \sigma_{y}^{(l)}\right) \\
& =\sum_{m=1}^{N} \frac{\epsilon_{m}}{2}\left(1+\sigma_{z}^{(m)}\right)-\frac{V}{2} \sum_{m<l=1}^{N}\left(\sigma_{x}^{(m)} \sigma_{x}^{(l)}+\sigma_{y}^{(m)} \sigma_{y}^{(l)}\right)
\end{aligned}
$$

where $\epsilon_{m}=\xi_{m}-V$.

In fact the spin analogy of the BCS Hamiltonian is well known and exact diagonalization of the pairing model in the spin space has been carried out in several previous works 15 . In this paper we propose a computational simulation method which is potential to realize in future with the development of quantum computer. The primary advantage of our method lies in the practical realization in experiments. Especially we can solve superconducting energy gap by this simulation method conveniently as following paragraphs. It is more practicably than other solution of energy gap, because it can simplify a $2^{N}$-dimension problem to an $N$-dimension problem. We know eigenvalues may not be solvable for high dimension matrix in principle. Now a $2^{N}$-dimension problem, exponential problem(EP) can be simplified an $N$-dimension problem, polynomial problem $(\mathrm{PP})$. In the following part we will describe how to transform EP to PP in detail.

Firstly the total Hamiltonian $H_{\text {spin }}$ will be expressed as the direct-sum of a set of submatrices ${ }^{5}$.

$$
H_{\text {spin }}=H_{s u b 0} \oplus H_{s u b 1} \oplus H_{s u b 2} \oplus \cdots \oplus H_{s u b N}
$$

The system states with the same spin-up state number form an absolute subspace. The subscripts $s u b 0, s u b 1, \cdots s u b N$ representing the number of the spin-up state in the corresponding subspace are respectively $0,1, \cdots, N$. Secondly we will prove that the eigenvalues of $H_{s u b 1}$ in sub1 submatrix justly are the eigenvalues of $H_{\mathrm{BCS}}$.

Note the $2^{N} \times 2^{N}$ operator as

$$
h(N, m)=\mathbf{I}^{\otimes(m-1)} \otimes\left(\begin{array}{ll}
1 & 0 \\
0 & 0
\end{array}\right) \otimes \mathbf{I}^{\otimes(N-m)} \quad(m=1,2, \cdots, N)
$$

whose $i$-th diagonal element is noted as $h(N, m)[i](i=1,2, \ldots, N)$. It is easy to see the non-diagonal elements of $h(N, m)$ are zero.

Lemma 1 For the Hamiltonian as $h(N, m)=\mathbf{I}^{\otimes(m-1)} \otimes\left(\begin{array}{ll}1 & 0 \\ 0 & 0\end{array}\right) \otimes \mathbf{I}^{\otimes(N-m)}$, the $\left(2^{N}-2^{N-i}\right)$-th element satisfies

$$
h(N, m)\left[2^{N}-2^{N-i}\right]=\delta_{i m} \quad(i, m=1,2, \cdots, N)
$$


Proof. We will prove this lemma by mathematic induction.

When $N=3$, it is easy to get three matrices, $m$ value is $1,2,3$ respectively.

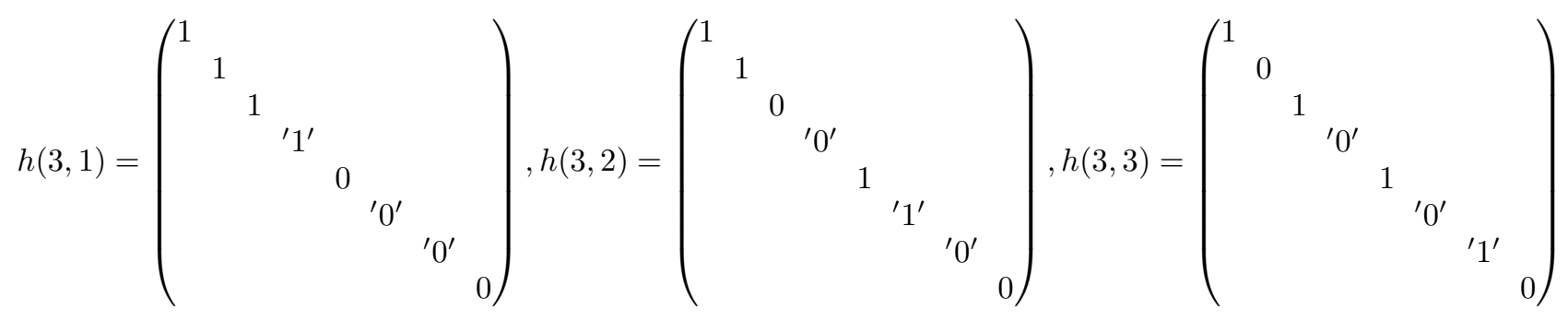

Here the non-diagonal elements are all 0 . These diagonal elements with' ' are $h(3, m)\left[2^{3}-2^{3-i}\right]$. It is easy to validate $h(3, m)\left[2^{3}-2^{3-i}\right]=\delta_{i m}$ and the last diagonal element $h(3, m)\left[2^{3}\right]$ is zero. If when $N=L$,

$$
h(L, m)\left[2^{L}-2^{L-i}\right]=\delta_{i m} \quad(i, m=1,2, \cdots, L)
$$

is right and

$$
h(L, m)\left[2^{L}\right]=0
$$

then we should examine whether $h(L+1, m)\left[2^{L+1}-2^{L+1-i}\right]=\delta_{i m}(i, m=1,2, \cdots, L+1)$ is right when $N=L+1$.

We will discuss it in two cases: $m \leqslant L$ and $m=L+1$.

(1) $m \leqslant L$ :

$$
\begin{aligned}
h(L+1, m) & =\mathbf{I}^{\otimes(m-1)} \otimes\left(\begin{array}{ll}
1 & 0 \\
0 & 0
\end{array}\right) \otimes \mathbf{I}^{\otimes(L+1-m)} \\
& =h(L, m) \otimes \mathbf{I}
\end{aligned}
$$

From Eq.(12) it is easy to see

$$
h(L+1, m)[2 x]=h(L+1, m)[2 x-1]=h(L+1, m)[x]
$$

so

$$
h(L+1, m)\left[2^{L+1}-2^{L+1-i}\right]=h(L, m)\left[2^{L}-2^{L-i}\right]=\delta_{i m} \quad(i=1,2, \cdots, L)
$$

Next we should also know the value of $h(L+1, m)\left[2^{L+1}-1\right]$. From Eq. (11) and Eq.(13) there is $h(L+1, m)\left[2^{L+1}-1\right]=$ $h(L, m)\left[2^{L}\right]=0$. From above discussion for $m \leqslant L(N=L+1)$, the equality $h(L+1, m)\left[2^{L+1}-2^{L+1-i}\right]=\delta_{i m}$ is valid.

(2) $m=L+1$ :

$$
\begin{aligned}
& h(L+1, L+1)=\mathbf{I}^{\otimes L} \otimes\left(\begin{array}{ll}
1 & 0 \\
0 & 0
\end{array}\right) \\
& =\left(\begin{array}{llll}
\left(\begin{array}{lll}
1 & 0 \\
0 & 0
\end{array}\right) & & & \\
& & \ddots & \\
& & & \left(\begin{array}{ll}
1 & 0 \\
0 & 0
\end{array}\right)
\end{array}\right)
\end{aligned}
$$

From the above expressions the $\left(2^{L+1}-1\right)$-th diagonal element $h(L+1, L+1)\left[2^{L+1}-1\right]=1$, that is to say

$$
h(L+1, L+1)\left[2^{L+1}-2^{L+1-i}\right]=1(i=L+1)
$$

And then for $i \neq L+1$,

$$
h(L+1, L+1)\left[2^{L+1}-2^{L+1-i}\right]=0
$$


because the even-th diagonal elements are all zero obviously. So for $m=L+1$ there is also $h(L+1, m)\left[2^{L}-2^{L-i}\right]=\delta_{i m}$. From the discussion (1) and (2) we have proved that when $N=L+1, h(L+1, m)\left[2^{L+1}-2^{L+1-i}\right]=\delta_{i m}(i, m=$ $1,2, \cdots, L+1)$ is valid. So for $\forall N \geqslant 3$ ( $N$ is the natural number), there is the equality

$$
h(N, m)\left[2^{N}-2^{N-i}\right]=\delta_{i m} \quad(i, m=1,2, \cdots, N)
$$

After the preparation we will prove that the eigenvalues of $H_{s u b 1}$ in sub1 subspace justly are the energy spectrum of quasiparticle excitation of $H_{\mathrm{BCS}}$. Firstly set a diagonal Hamiltonian as $H^{\mathrm{diag}}=\frac{1}{2} \sum_{m=1}^{N} E_{m}\left(\gamma_{m}^{\dagger} \gamma_{m}+\gamma_{-m}^{\dagger} \gamma_{-m}\right), \gamma_{m}^{\dagger} \gamma_{m}$ and $\gamma_{-m}^{\dagger} \gamma_{-m}$ are the quasiparticle number operators. According to the previous analogy rule of number operators Eq.(6) the spin-analogy form of $H^{\text {diag }}$ is

$$
H_{\text {spin }}^{\text {diag }}=\sum_{m=1}^{N} E_{m} h(N, m)
$$

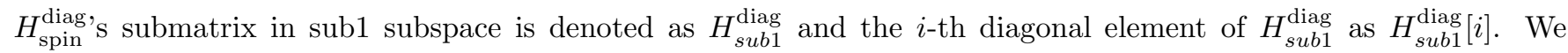
can find the $i$-th diagonal element of $H_{s u b 1}^{\text {diag }}$ is the $\left(2^{N}-2^{N-i}\right)$-th diagonal element of the total Hamiltonian $H_{\mathrm{spin}}^{\text {diag }}$. According to Eq. (9)

$$
\begin{aligned}
H_{\text {subi } 1}^{\text {diag }}[i] & =H_{\mathrm{spin}}^{\mathrm{diag}}\left[2^{N}-2^{N-i}\right] \\
& =\sum_{m=1}^{N} E_{m} h(N, m)\left[2^{N}-2^{N-i}\right] \\
& =\sum_{m=1}^{N} E_{m} \delta_{i m} \\
& =E_{i}
\end{aligned}
$$

So the spin-analogy Hamiltonian of a diagonal BCS Hamiltonian $H^{\text {diag }}$ in sub1 subspace has the same eigenvalues as the diagonal BCS Hamiltonian's energy spectrum. We can deduce further that the eigenvalues of $H_{s u b 1}$ are justly the eigenvalues of $H_{\mathrm{BCS}}$ whether which is diagonal or not, because $H_{\mathrm{BCS}}$ can be written as the diagonal form like $H^{\text {diag }}$ generally, i.e. $H_{\mathrm{BCS}} \longrightarrow \frac{1}{2} \sum_{m=1}^{N} E_{m}\left(\gamma_{m}^{\dagger} \gamma_{m}+\gamma_{-m}^{\dagger} \gamma_{-m}\right)+\cdots$, while we need not care about how to obtain the diagonal form Hamiltonian. Consequently it implies that if we have diagonalized $H_{s u b 1}$ we can get the energy spectrum of quasiparticle excitation of $H_{\mathrm{BCS}}$ and get energy gap further avoiding complex computation. One of the classical methods to diagonalize $H_{\mathrm{BCS}}$, Bogoliubov transformation method is available under the mean-field approximation, which is not exact especially in the case of limited $N$. Another famous method about the exact solution of BCS Hamiltonian has been proposed in the 60's by Richardson ${ }^{4}$. He considered the system with $M \leqslant N$ pairs electrons and constructed a set of operators $\mathbf{R}_{j}(j=1, \cdots, N)$ commuted with $H_{\mathrm{BCS}}$, finally gave the expression of the eigenvalues $\lambda_{j}$ of $\mathbf{R}_{j}$ through solving the $M$ coupled algebraic equations. $\lambda_{j}$ is not yet the eigenvalue of $H_{\mathrm{BCS}}$. We can more directly and simply give the energy spectrum of $H_{\mathrm{BCS}}$.

Thus it can be seen the idea of diagonalizing $H_{s u b 1}$ instead of solving the eigenvalues of $H_{\mathrm{BCS}}$ directly is better than those classical ones. Now a key problem that how to get the submatrix $H_{s u b 1}$ is placed to the front. The correlative work $^{16}$ in our group has proved that the general form of $H_{s u b 1}$ :

$$
H_{s u b 1}[i, i]=\epsilon_{i}, \quad H_{s u b 1}[i, j]=-V \quad(i, j=1,2, \cdots, N ; i \neq j)
$$

$H_{s u b 1}[i, j]$ is the matrix element of $H_{s u b 1}$.

Finally it is necessary to check the methods in numerical computation. Here we will compare our solution with the result of the mean-field approximation by the value of superconducting energy gap $\Delta(T=0 K)$. According to the physics meaning of $\Delta$, the energy required to excite at least a quasiparticle from the Fermi surface, the energy of the element excitation is written as $E_{m}=\left(\xi_{m}^{2}+\Delta^{2}\right)^{1 / 2} \underline{\underline{3}}$ In fact the element excitation energy is also the eigenvalue of $H_{\mathrm{BCS}}$. After getting the eigenvalue of $H_{s u b 1}$, i.e. the eigenvalue of $H_{\mathrm{BCS}}$, we can get the value of $\Delta$ by solving equation $E_{m}=\left(\xi_{m}^{2}+\Delta^{2}\right)^{1 / 2}$. But in order to get rid of the effect of energy zero the equation

$$
\left(\xi_{1}^{2}+\Delta^{2}\right)^{1 / 2}-\left(\xi_{2}^{2}+\Delta^{2}\right)^{1 / 2}=E_{1}-E_{2}
$$


is used to solve $\Delta$ in practice, because the difference of eigenvalues dosen't depend on the energy zero and that we find the energy difference between the ground and the first excited state $E_{2}-E_{1}$ is by far larger than $E_{3}-E_{2}, E_{4}-$ $E_{3}, \cdots, E_{N}-E_{N-1}$ in the course of the numerical computation. Here $\xi_{1}<\xi_{2}<\cdots<\xi_{N}$ and $E_{1}<E_{2}<\cdots<E_{N}$.

The another kind of solution used in comparing is the following energy gap equation ${ }^{12}$ :

$$
1=\frac{1}{2} V \sum_{m} \frac{1}{\sqrt{\xi_{m}^{2}+\Delta^{2}}}
$$

Here we consider the reduced BCS model whose energies are given for simplicity by $\xi_{m}=m \delta \frac{17}{18}, 19$, here $\delta$ is the average level spacing which is inversely proportional to the size of the grains. In the strong coupling regime, corresponding to large grains or strong coupling constants, $\delta \ll \Delta$. In the weak coupling region, corresponding to small grains or small coupling constants, $\delta \gg \Delta^{17}$. From much research about ultrasmall superconducting grains ${ }^{20}$, the mean-field theory is not suitable in the weak coupling region. It has been proved that the corrections to the mean-field results are small in large grains become important in the opposite limit ${ }^{18}$. So in this paper we carry out the numerical computation in the first case, $\delta \ll \Delta$. We also take the coupling constants $V=\lambda \delta$ in order to discuss conveniently. In order to give the numerical pictures, we suppose the value of $V$ by the rough estimate. From BCS theory, the Cooper pair lies in the attraction area, i.e. $0 \leq \xi_{m} \leq \hbar \varpi_{D}{ }^{12}$. For metal Debye energy $\hbar \varpi_{D} \sim 10^{-2} \mathrm{eV}$, we can set $V \sim 2 \times 10^{-6} \mathrm{eV}$ by rough estimate. The estimate process is put to the later appendix. Another two variables $\lambda$ and $N$ are taken as the independent variables of the energy gap.

We list our results in diagrams. In FIG 1 setting $\lambda=10$, the energy gap is plotted as the function of energy level number, which is the mono-increasing function of the energy level number $N$.

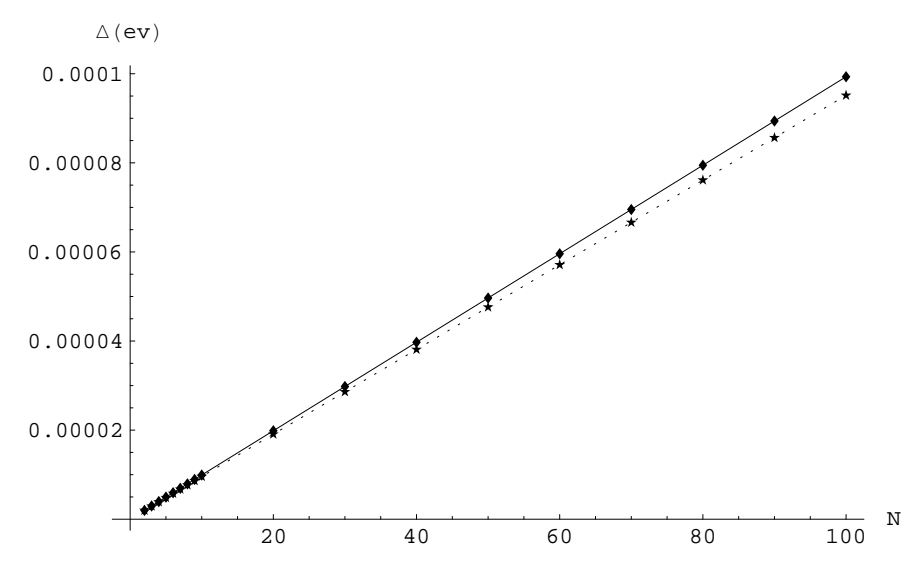

FIG. 1: The energy gap $\Delta$ as a function of the number of energy level, $N$. We give the comparison between two results by the different methods. The solid line is the solution of the energy-gap equation; the dashed line is our result by spin analogy and diagonalizing submatrix. Here we choose $V \sim 2 \times 10^{-6} \mathrm{eV}, \lambda=10$.

The relative error between our result and that of the Eq. (16) is not more than $5 \%$ in the range from $N=2$ to $N=100$ and fixed $\lambda(=10)$. It shows that the result from our method is well consistent with the solution of the energy gap equation. In order to check the universality of this new method, we also give the dependence relation between the energy gap and the level spacing, see FIG 2

It is clear to see $\Delta$ changes gently with $\lambda$ when $\lambda$ is large enough. That is to say, $\Delta$ is almost independent of $\delta$ when $\delta$ is small enough. It shows the rationality of $\delta \ll \Delta$ on the inverse hand. Obviously, in FIG 2 , when $\lambda$ is larger than 80 , the relative error is less than $1.1 \%$.

We also consider a small departure from the fermi surface, that is $\xi_{m}=\xi_{0}+m \delta$. $\xi_{0}$ is a small value, $\xi_{0} \sim \delta$. In the following discussion we note $\xi_{0}=b \delta, b$ is the natural number. The small departure from the fermi surface reduces the energy gap and energy gap is not a real root when the departure reaches a critical value, see FIG 3

According to above comparison we know two results are consistent well, while our method to solve the energy spectrum doesn't include approximation, which indicates that our result includes that obtained by mean field theory and is superior to it.

In summary, we have proposed an exact numerical simulation method to calculate the energy spectrum of the reduced BCS Hamiltonian by spin analogy and diagonalizing submatrix. A numerical computation to verify the validity of our computational method is given. We make a comparison between our method and energy gap equation Eq.(16), and two results are well consistent in numerical computation. By examining the change of the energy gap 


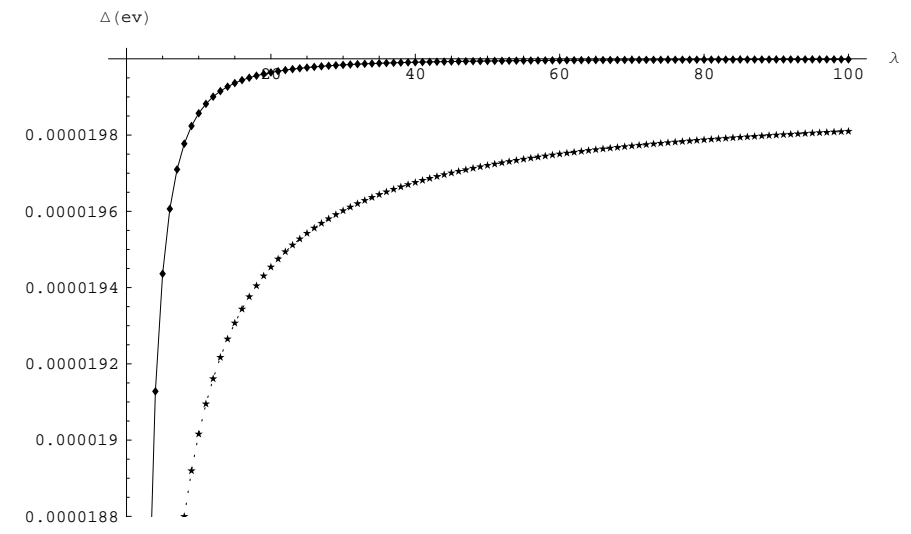

FIG. 2: When $N=20$, Energy gap $\Delta$ is plotted as the function of $\lambda$. We give the comparison between two results: the solid line is the solution of the energy-gap equation; the dashed line is our result by spin analogy and diagonalizing submatrix. Here we choose $V \sim 2 \times 10^{-6} \mathrm{eV}$.

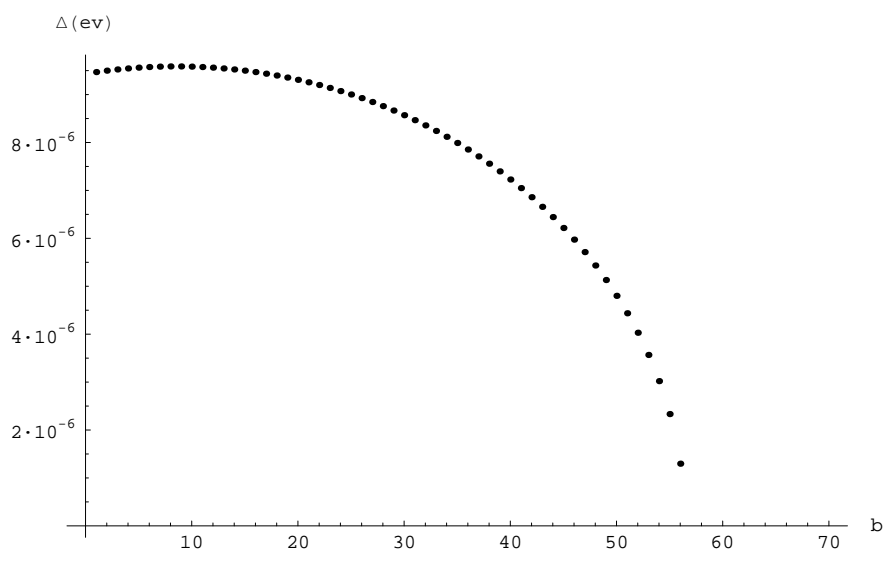

FIG. 3: When $V \sim 2 \times 10^{-6} \mathrm{eV}, \lambda=10, N=10$ the energy gap $\Delta$ is ploted as the function of $b$ through the quantum simulation. When $b>56$, there is not the real root for $\Delta$. The result is obtained by the quantum simulation. Similar result will be obtained by the energy gap equation Eq. (16).

value under the change of the parameter, we include the excellent consistency between the two results by the different methods is independent on the particular parameter. It implies that one can implement this quantum simulation on a quantum computer and the result will be believable. Currently a new experiment about 2-qubit simulation of the pairing Hamiltonian on an NMR quantum computer has been realized and get the energy spectrum of the pairing Hamiltonian successfully $\stackrel{10}{ }$. With the development of quantum computer, especially the manipulation and control of multi-qubit system, this new simulation computation method has the great potential in practical application.

We are grateful Xiaosan Ma, WanQing Niu, Zhao Ningbo, Zhu Rengui and Su Xiao-Qiang for helpful discussion. This work was founded by the National Fundamental Research Program of China with No. 2001CB309310, partially supported by the National Natural Science Foundation of China under Grant No. 60173047 and the Natural Science Foundation of Anhui Province.

\section{APPENDIX A: APPENDIX: ESTIMATE ABOUT $V$}

For metal element $g(0) V \approx 0.2 \sim 0.3 \frac{12}{2}, g(0)$ is the state density which have some spin directions on the Fermi surface.

$$
\int_{0}^{\infty} 2 g(\varepsilon) d^{3} \varepsilon=1
$$


According to the assumption

$$
\begin{aligned}
& g(\varepsilon)=0, \quad \text { if } \varepsilon>\hbar \varpi_{D} \\
& g(\varepsilon)=g(0), \quad \text { if } \varepsilon \leq \hbar \varpi_{D}
\end{aligned}
$$

formalism (A1) can be written as

$$
\int_{0}^{\hbar \varpi_{D}} 2 g(0) d^{3} \varepsilon=1
$$

so from BCS theory, the Cooper pair lies in the attraction area, ie $0 \leq \xi_{k} \leq \hbar \varpi_{D}$. On substitution of $\hbar \varpi_{D} \sim 10^{-2} \mathrm{eV}^{12}$ we can estimate $g(0) \approx 10^{5}$, so $V \sim 2 \times 10^{-6} \mathrm{eV}$.

1 J. Bardeen, L. N. Cooper, and J. R. Schrieffer, Phys. Rev. 108, 1175 (1957).

2 Neil W. Ashcroft and N. David Mermin, Solid State Physics (Holt, Rinehart and Winston, 1976)

3 Philip L. Taylor and Olle Henonen, A Quantum Approach to Condensed Matter Physics, Cambridge (2002)

${ }^{4}$ R. W. Richardson, Phys. Lett. 3, 108 (1963), R. W. Richardson, Phys. Lett. 8, 277 (1963)

5 L.-A. Wu, M. S. Byrd, and D. A. Lidar, Phys Rev Lett. 89, 057904 (2002); J. Dukelsky, J. M. Román, and G. Sierra, Phys Rev Lett. 90, 249803 (2003); L.-A. Wu, M. S. Byrd, and D. A. Lidar, Phys Rev Lett. 90, 249804 (2003)

6 R. P. Feynman, Int. J. theor. Phys. 21, 467 (1982), A. Galindo and M. A. Martín-Delgado, Rev. Mod. Phys. 74, 347 (2002)

7 S. Somaroo, C.H. Tseng, T. F. Havel, R. Laflamme, and D. G. Cory, Phys. Rev. Lett. 82,5381 (1999)

8 C.H. Tseng, S. Somaroo, Y. Sharf, E. Knill, R. Laflamme and etc. Phys. Rev. A 61, 032309 (2000)

9 A. K. Khitrin and B. M. Fung, Phys. Rev. A 64, 032306 (2001)

10 Xiao-dong Yang, An Min Wang, Feng Xu and Jiang-Feng Du, quant-ph/0410143

11 An Min Wang and Xiao-dong Yang, quant-ph/0410007

12 Zheng-zhong Li, The theory of Solid State Physics, in Chinese (2002)

13 P. G. de Gennes, Superconductivity of Metals and Alloys, Addison-Wesley (1989); Gerald D. Mahan, Many-Particle Physics, second edition, Plenum Press, New York (1990)

14 Philip B. Allen, in Concise Encyclopedia of Solid State Physics, edited by Rita G. Lerner and George L.Trigg (AddisonWesley Publishing Company, 1983), p. 266

15 Alexander Volya, B. Alex Brow, Vladimir Zelevinsky, Phys. Lett. B. 509, 37 (2001);

16 An Min Wang and F Xu, cond-mat/0409400

17 J. Dukelsky and G. Sierra, Phys. Rev. Lett. 83, 172 (1999)

18 K. A. Matveev and A. I. Larkin, Phys. Rev. Lett. 78 , 3749 (1997)

19 A. Mastellone et al., Phys. Rev. Lett. 80, 4542 (1998)

20 J. von Delft and D.C. Ralph, Phys. Report. 345, 61-173 (2001) 